\title{
基于分数阶微分的重叠峰分辨方法
}

\author{
李远禄* 于盛林 郑 罡
}

(南京航空航天大学自动化学院, 南京 210016)

\begin{abstract}
摘要根据特定峰信号微分的极值和过零点随着微分阶次的变化而改变, 建立了峰信号的极 值和过零点与其相应微分阶次的关系式, 进而得到了两类参数估计器, 利用这些估计器可以估计 出 Gaussian 峰、Lorentzian 峰和 Tsallis 峰信号的特征参数. 首先, 利用分数阶微分器获得特定峰 信号的分数阶微分; 其次, 根据峰信号的极值与其微分阶次的关系式和过零点与其微分阶次的关 系式, 建立了估计器 I 和估计器 II; 再利用这些估计器便可以提取峰信号的特征参数; 最后, 用 Tsallis 峰作为子峰模型对未知重叠峰信号进行分辨. 仿真和实测重叠峰信号的分辨实验表明本方 法简单高效.
\end{abstract}

\section{关键词 分数阶微分滤波器 分数阶微分 重叠峰分辨}

\section{1 引言}

在化学领域, 重叠的化学信号是比较常见的, 如 重叠的色谱峰、重叠的伏安峰和重叠的光谱信号等. 由于重叠现象的存在, 给进一步的定性和定量分析 带来了困难. 为了提高原有信号的分辨率，使其能用 于定性和定量分析, 借助于信号处理方法来分辨重 叠峰是一个重要的研究方向. 传统上, 对重叠峰信号 进行分辨分为两个步骤: (1) 确定重叠峰中各子峰的 位置, 主要方法有导数分峰法 ${ }^{[1]}$ 、Fourier去卷积 法 $[2,3]$ 、微分消卷积法 $[4,5]$ 、小波分析及其改进法 ${ }^{[6 ~ 10]}$ 等. 这些方法能提高重叠峰中各子峰的分辨能力, 但不 能彻底形成分峰, 也不能得到各子峰的参数 $[11]$. (2) 重叠峰信号的分解, 目的在于获取重叠峰中各子 峰的信息, 即将提高分辨能力后的峰信号进一步分 解成独立的子峰. 一般采用高斯(Gaussian)峰、洛伦 兹(Lorentzian) 峰或这两种峰以不同的比例组合作为 模型来拟合需要分解的重叠峰信号, 得到各子峰的 形状、位置和面积等信息, 通常称作曲线拟合法 ${ }^{[12]}$.

本文借助于分数阶微分这一新的数学工具, 利
用重叠峰信号的分数阶微分的过零点和极值与其微 分阶次的关系, 建立了一种新的重叠峰分辨方法, 将 传统峰分辨中的确定子峰位置和分解子峰两个步骤 合二为一, 简化了重叠峰信号分辨过程. 采用分数阶 微分作为处理工具, 既保留了导数分峰法的优点, 还 可降低噪声的影响. 可广泛应用于多种重叠峰信号 分辨, 通过仿真和实测信号分析, 该方法简单高效.

\section{2 理论部分}

\section{1 分数阶微分滤波器}

分数阶微分的计算常用数值算法, 一种最基本 的离散形式是Grunwald和Letnikov公式 ${ }^{[13]}$ :

$$
{ }_{c} D_{x}^{\alpha} f(t)=\lim _{h \rightarrow 0} \frac{1}{h^{\alpha}} \sum_{j=0}^{[(t-c) / h]}(-1)^{j}\left(\begin{array}{l}
\alpha \\
j
\end{array}\right) f(t-j h),
$$

式中 $\left(\begin{array}{c}\alpha \\ j\end{array}\right)$ 为二项式系数, $h$ 为离散步长. 在信号处理中, 一般采用有限冲击响应(FIR)滤波器来实现.

定义 1: 信号 $f(t)$ 经有限冲击响应(FIR)滤波器 
的 $\left(\alpha \in R^{+}\right)$阶微分输出为

$$
y=\frac{1}{h^{\alpha}} \sum_{j=0}^{[(t-c) / h]} b_{j}^{\alpha} f(t-j h),
$$

式中

$$
b_{j}^{\alpha}= \begin{cases}1, & j=0 \\ (-1)^{n} \frac{\alpha(\alpha-1)(\alpha-2) \ldots(\alpha-j+1)}{j !}, & j>0 .\end{cases}
$$

\subsection{3 种峰信号的分数阶微分}

一阶导数和二阶导数已广泛用于峰信号位置的 检测和重叠峰信号分析, 文献[1]曾采用 2.5 阶导数分 辨重叠峰信号, 取得了较好的效果, 然而, 它只是用 来检测峰的位置, 本文通过深入研究发现, Gaussian 峰信号、Lorentzian峰信号和Tsallis峰信号, 它们的极 值和过零点会随着微分阶次的变化而改变, 通过建 立这些峰信号的极值和过零点与其相应微分阶次的 关系式, 进而得到两类参数估计器, 利用这些估计器 可以估计出这些峰信号的特征参数, 为定量分析重 叠峰信号提供了一种新的方式.

3 种峰信号分别为 Gaussian 峰 $f_{1}(t)=A e^{\left[-(t-\mu)^{2} /\left(2 \sigma^{2}\right)\right]}$, 式中 $A=3.0, m=1.0, \sigma=0.8,-4 \leqslant t \leqslant 6$; Lorentzian 峰: $f_{2}(t)=A \sigma^{2} /\left[(t-\mu)^{2}+\sigma^{2}\right]$, 式中 $A=4.0, \mu=1.0, \sigma=1.0$, $-4 \leqslant t \leqslant 6$; Tsallis 峰: $f_{3}(t)=A\left[1+\frac{q-1}{3-q} \frac{(t-\mu)^{2}}{\sigma^{2}}\right]^{\frac{-1}{q-1}}$, 式中 $A=4.0, \mu=1.0, \sigma=1.0, q=2.5,-4 \leqslant t \leqslant 6$. 首先, 对 3 种峰信号进行离散得到离散序列 $f_{1}(n), f_{2}(n)$ 和 $f_{3}(n)$, 然后利用分数阶微分滤波器获得 3 种峰信号 的不同分数阶微分. 图 1 给出了 Gaussian 峰、 Lorentzian 峰、Tsallis 峰的分数阶 $(1 \leqslant \alpha \leqslant 2)$ 微分, 从 图 1 中可看出, 3 种峰信号的极值和过零点会随着微 分阶次 $\alpha$ 的改变而改变.

\subsection{3 种峰信号的参数估计器的建模}

首先，根据特定峰信号的分数阶微分的过零点 与其相应微分阶次的关系建立了一类参数估计器 I ; 然后，根据特定峰信号的分数阶微分的极值与其微 分阶次的关系建立了另一类参数估计器 II , 在这两 类参数估计器中, 有一个共同的参数: 峰宽 $\sigma$, 对于 待处理的未知重叠峰, 正是利用两种估计器的峰宽 一致来确定分峰的有效性.

\subsection{1 关于过零点的参数估计器}

用数值方法分别求取 3 种峰信号的 $\alpha(1 \leqslant \alpha \leqslant 2)$ 阶微分的过零点, 再用二次多项式拟合 3 种峰信号的 过零点与微分阶次的关系，其表达式为：

$$
x_{0}(\alpha)=c_{2} \alpha^{2}+c_{1} \alpha+c_{0},
$$

式中 $x_{0}(\alpha)$ 表示 3 种峰信号的 $\alpha$ 阶导数的过零点, $c_{0}, c_{1}$, $c_{2}$ 为二次多项式的系数. 图 2 给出了 3 种峰信号的过 零点与其微分阶次之间的关系. 实线表示二次多项 式拟合结果，星号表示峰信号的 $\alpha$ 阶导数的实际计算 得到的过零点值.

经计算得 Gaussian 峰的一阶和二阶微分的过零 点分别为

$$
x_{0}(1)=\mu ; x_{01}(2)=\mu-\sigma ; x_{02}(2)=\mu+\sigma .
$$

将 $x_{0}$ (1) 和 $x_{01}$ (2) 代入式(3)可得 Gaussian 峰的两个 参数 $\mu$ 和 $\sigma$ 的估计器 I 为

$$
\left\{\begin{array}{l}
\tilde{\mu}=c_{2}+c_{1}+c_{0} \\
\tilde{\sigma}=-3 c_{2}-c_{1} .
\end{array}\right.
$$

经计算得 Lorentzian 峰的一阶和二阶微分的过零 点分别为

$$
x_{0}(1)=\mu ; x_{01}(2)=\mu-\sigma / \sqrt{3} ; x_{01}(2)=\mu+\sigma / \sqrt{3} \text {. }
$$
将 $x_{0}$ (1) 和 $x_{01}$ (2) 代入式(3)可得 Lorentzian 峰的两个 参数 $\mu$ 和 $\sigma$ 的估计器 $\mathrm{I}$ 为
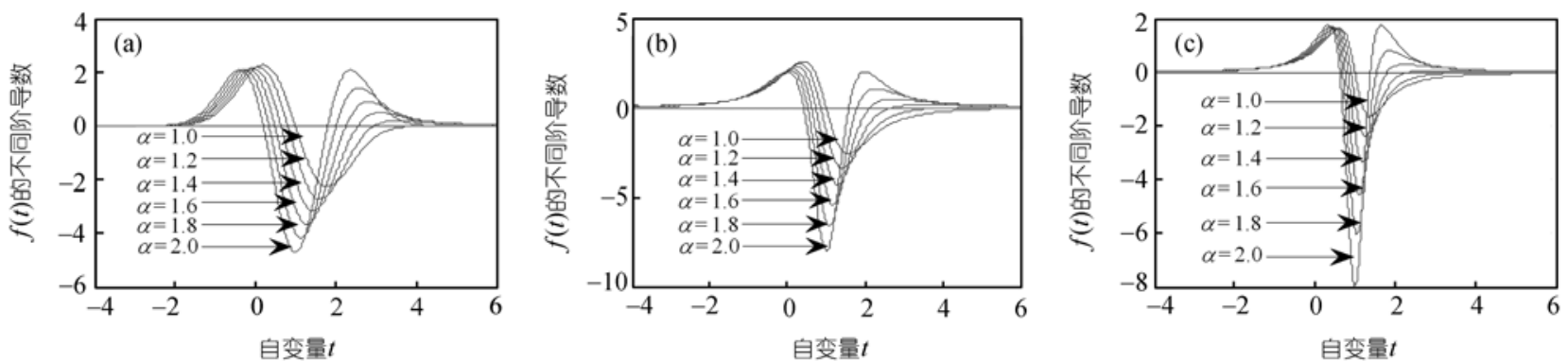

图 1 Gaussian 峰(a)、Lorentzian 峰(b)和 Tsallis 峰(c)的 $\alpha$ 阶 $(1 \leqslant \alpha \leqslant 2)$ 微分 

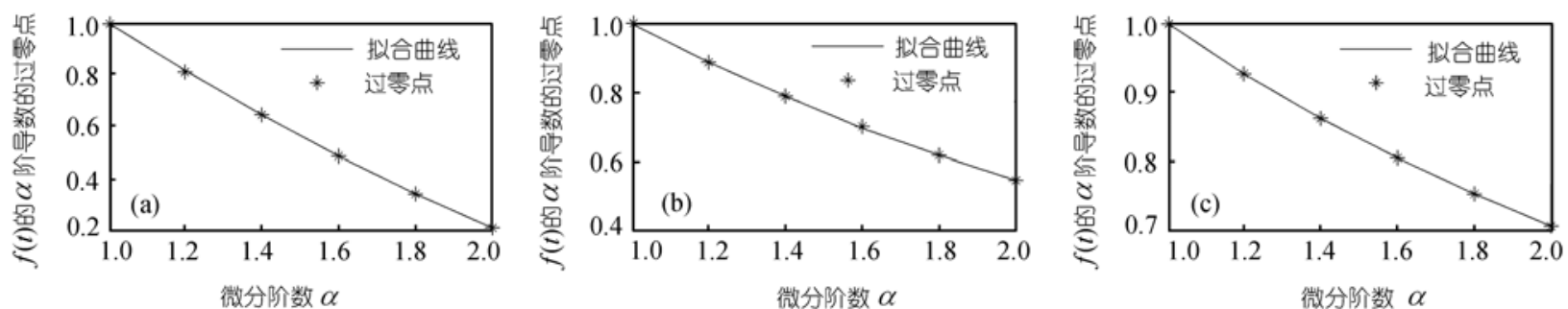

图 23 种峰信号的 $\alpha$ 阶微分的过零点与其微分阶次 $\alpha$ 之间的关系

(a) Gaussian 峰 $\alpha$ 阶微分的过零点与其微分阶次之间的关系; (b) Lorentzian 峰 $\alpha$ 阶微分的过零点与其微分阶次之间的关系;

(c) Tsallis 峰 $\alpha$ 阶微分的过零点与其微分阶次之间的关系

$$
\left\{\begin{array}{l}
\tilde{\mu}=c_{2}+c_{1}+c_{0} \\
\tilde{\sigma}=-\sqrt{3}\left(3 c_{2}+c_{1}\right) .
\end{array}\right.
$$

经计算得 Tsallis 峰的一阶和二阶微分的过零点分 别为

$$
\begin{aligned}
& x_{0}(1)=\mu ; \\
& x_{01}(2)=\mu-\frac{\sqrt{-q^{2}+2 q+3}}{1+q} \sigma ; \\
& x_{02}(2)=\mu+\frac{\sqrt{-q^{2}+2 q+3}}{1+q} \sigma .
\end{aligned}
$$

将 $x_{0}(1)$ 和 $x_{01}$ (2) 代入式(3)可得 Tsallis 峰的两个参数 $\mu$ 和 $\sigma$ 的估计式 I :

$$
\left\{\begin{array}{l}
\tilde{\mu}=c_{2}+c_{1}+c_{0} \\
\tilde{\sigma}=-\frac{(q+1)}{\sqrt{\left(-q^{2}+2 q+3\right)}}\left(3 c_{2}+c_{1}\right) .
\end{array}\right.
$$

3 种峰信号的二阶微分有两个过零点, 这里选择 $x_{01}(2)$ 来建立参数估计器, 对于单峰信号也可选择 $x_{02}(2)$ 来建立参数估计器, 但对于重叠峰信号, 从左 至右分辨重叠峰信号时, 我们选择最左边的过零点 来建立参数估计器.

\subsection{2 关于最大值的参数估计器}

分别求取 3 种峰信号的 $\alpha$ 阶 $(1 \leqslant a \leqslant 2)$ 微分的最 大值，再用二次多项式拟合 3 种峰信号的极值与其相 应的微分阶次 $\alpha$ 之间的关系，其表达式为

$$
F_{\max }(\alpha)=d_{2} \alpha^{2}+d_{1} \alpha+d_{0} .
$$

式中 $F_{\text {max }}(\alpha)$ 表示 3 种峰信号的 $\alpha$ 阶导数的最大值, $d_{0}$, $d_{1}, d_{2}$ 为二次多项式的系数, $\alpha$ 为微分的阶次. 图 3 给 出了 3 种峰信号的最大值与其微分阶次之间的关系. 图 3 中实线表示二次多项式拟合结果，星号表示峰信 号的 $\alpha$ 阶微分的实际计算得到的最大值.

通过计算得到 3 种峰信号的一阶、二阶微分的最 大值, 对于 Gaussian 峰有:

$$
\left\{\begin{array}{l}
F_{\text {max }}(1)=A \sigma^{-1} e^{-1 / 2} \\
F_{\text {max }}(2)=2 A \sigma^{-2} e^{-3 / 2} .
\end{array}\right.
$$

将式 $F_{\text {max }}(1)$ 和 $F_{\text {max }}$ (2) 代入式(10)可得 Gaussian 峰的 两个参数 $A$ 和 $\sigma$ 的估计器 II :

$$
\tilde{A}=\frac{2\left(d_{2}+d_{1}+d_{0}\right)^{2}}{e^{1 / 2}\left(4 d_{2}+2 d_{1}+d_{0}\right)}, \tilde{\sigma}=\frac{2\left(d_{2}+d_{1}+d_{0}\right)}{e\left(4 d_{2}+2 d_{1}+d_{0}\right)} .
$$

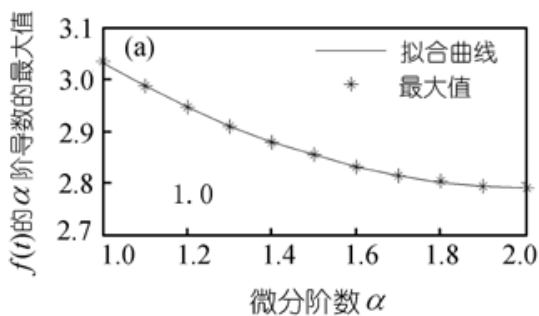

微分阶数 $\alpha$

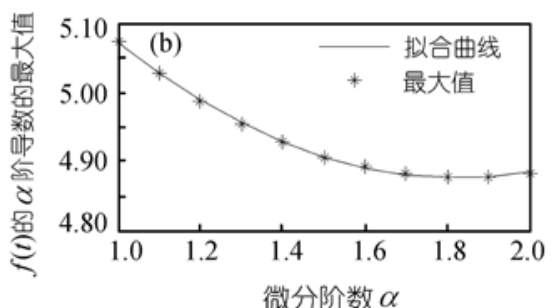

微分阶数 $\alpha$

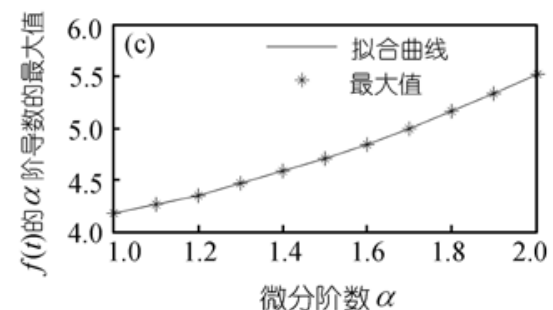

微分阶数 $\alpha$

图 33 种峰信号的 $\alpha$ 阶微分的最大值与其微分阶次 $\alpha$ 之间的关系 (a) Gaussian 峰 $\alpha$ 阶微分的最大值与其微分阶次 $\alpha$ 之间的关系; (b) Lorentzian 峰 $\alpha$ 阶微分的最大值与其微分阶次 $\alpha$ 之间的关系; 
对于 Lorentzian 峰有:

$$
\left\{\begin{array}{l}
F_{\max }(1)=(3 / 4)^{3 / 2} A \sigma^{-1} \\
F_{\text {max }}(2)=A \sigma^{-2} / 2 .
\end{array}\right.
$$

将式 $F_{\text {max }}(1)$ 和 $F_{\text {max }}(2)$ 代入式(10) 可得Lorentzian 峰 的两个参数 $A$ 和 $\sigma$ 的估计器 II :

$$
\left\{\begin{array}{l}
\tilde{A}=\frac{4^{5 / 2}\left(d_{2}+d_{1}+d_{0}\right)^{2}}{3^{3}\left(4 d_{2}+2 d_{1}+d_{0}\right)} \\
\tilde{\sigma}=\frac{d_{2}+d_{1}+d_{0}}{\sqrt{27 / 16}\left(4 d_{2}+2 d_{1}+d_{0}\right)} .
\end{array}\right.
$$

对于 Tsallis 峰有:

$$
\left\{\begin{array}{l}
F_{\max }(1)=2 A \sigma^{-1}\left(2 q+3-q^{2}\right)^{-1 / 2}\left(\frac{2 q}{q+1}\right)^{\frac{-q}{q-1}} \\
F_{\max }(2)=4 A \sigma^{-2}(3-q)^{-1}\left(\frac{4 q-2}{q+1}\right)^{\frac{-(2 q-1)}{q-1}} .
\end{array}\right.
$$

将 $F_{\text {max }}(1)$ 和 $F_{\text {max }}$ (2) 代入式(10)可得 Tsallis 峰的两个 参数 $A$ 和 $\sigma$ 的估计器 II :

$$
\left\{\begin{array}{l}
\tilde{A}=\frac{\left(d_{2}+d_{1}+d_{0}\right)^{2}\left(\frac{2 q}{q+1}\right)^{\frac{2 q}{q-1}}\left(2 q+3-q^{2}\right)}{\left(4 d_{2}+2 d_{1}+d_{0}\right)(3-q)\left(\frac{4 q-2}{q+1}\right)^{\frac{2 q-1}{q-1}}} \\
\tilde{\sigma}=\frac{2\left(d_{2}+d_{1}+d_{0}\right)\left(\frac{2 q}{q+1}\right)^{\frac{q}{q-1}} \sqrt{2 q+3-q^{2}}}{\left(4 d_{2}+2 d_{1}+d_{0}\right)(3-q)\left(\frac{4 q-2}{q+1}\right)^{\frac{2 q-1}{q-1}}} .
\end{array}\right.
$$

原则上, 也可选择极小值来建立参数估计器, 但对于 重叠峰信号, 从左至右分辨重叠峰时, 我们选择最左 边的极值.

\section{3 实验部分 ${ }^{[14]}$}

\section{1 仪器}

LK98 微机电化学分析系统(天津市兰力科化学 电子高技术有限公司研制), 三电级体系, 采用悬录 电极为工作电极, $\mathrm{Ag} / \mathrm{AgCl}(0.1 \mathrm{~mol} / \mathrm{L} \mathrm{KCl})$ 为参比电极. 铂电极为辅助电极.

\section{2 试剂}

所用试剂均为分析纯, 配制试液所用水为二次 去离子水. Cd (II) 和 In (III)标准溶液的浓度均为
$1.0 \times 10^{-2} \mathrm{~mol} / \mathrm{L}$. 采用 $0.1 \mathrm{~mol} / \mathrm{L} \mathrm{KNO}_{3}$ 作为支持电解质.

\section{3 实验方法}

分别取一定量标准溶液, 配制成 Cd (II) (浓度为 $1.0 \times 10^{-5} \mathrm{~mol} / \mathrm{L}$ ) 和 In (III) (浓度为 $2.5 \times 10^{-5} \mathrm{~mol} / \mathrm{L}$ ) 的混 合溶液, 并移入电解池中. 底液为 $0.1 \mathrm{~mol} / \mathrm{L}$ 的 $\mathrm{KNO}_{3}$. 实验前向混合溶液中通入高纯氮 $20 \mathrm{~min}$ 以除去氧气, 并保持温度在 $20^{\circ} \mathrm{C}$ 左右. 在 LK-98 电分析系统中以 方波伏安法对混合溶液进行测定. 实验条件：扫描范 围为 $-0.8 \sim-0.4 \mathrm{~V}$, 扫描增量为 $1 \mathrm{mV}$, 方波幅度和方 波周期分别为 $25 \mathrm{mV}$ 和 $0.16 \mathrm{~s}$. 将记录的数据输入微 机进行处理.

\section{4 结果分析与讨论}

本节主要检验 2.3 节所建立的参数估计器在分辨 重叠峰信号时的效果, 其方法是用 Tsallis 峰作为子峰 模型, 然后通过扫描 Tsallis 峰参数 $q$ 来确定各子峰的 参数, 即通过扫描估计器式(9)和估计器式(16)中的 $q$, 选取两个估计器所得参数 $\tilde{\sigma}$ 的差最小的 $q$ 作为 Tsallis 峰参数 $q$ 的值, 对应此 $q$ 值的估计值 $\tilde{A}, \tilde{\sigma}, \tilde{\mu}$ 为相应 Tsallis 峰参数的估计值. 其步骤是从左至右分辨重叠 峰信号, 首先用分数阶微分滤波器对重叠峰进行滤 波, 获得相应微分阶次的过零点和极值, 然后根据估 计器 I 和 II 进行参数估计, 从而得到重叠峰信号中 的一个子峰, 最后, 将此子峰从重叠峰信号中减掉, 重复上述过程, 直到所有的峰信号处理完备. 尽管所 给仿真峰信号均为连续信号, 但在实际处理时都需 要进行离散, 事实上所处理的信号均为离散信号. 因 此本文方法可直接应用于离散信号处理.

仿真重叠峰信号如图 4 所示. 这些重叠峰信号是 由 Gaussian 峰和 Lorentzian 峰合成, 图 4(a)分离度为 0.5 , (b)分离度为 0.75 , (c)分离度为 1.0 , 图中 G 峰代表 Gaussian 峰, L 峰代表 Lorentzian 峰, 其他参数见表 1 处理前的数据, 各子峰参数的估计结果及其相对误 差见表 1 . 从表 1 可看出, 参数估计误差不但与分离 度有关, 还与峰高比和峰宽比有关.

\section{1 各种参数的变化对参数估计结果的影响}

以 Gaussian 和 Lorentzian 混合双峰为例, Gaussian 峰参数 $\mu=-1.0, A=20, \sigma=1$ 和 Lorentzian 峰参数 $\mu 1=$ 1.5, $\sigma 1=0.5, A 1$ 由峰高比确定, 考察峰高比对参数估 计误差的影响，其结果见图 5. 

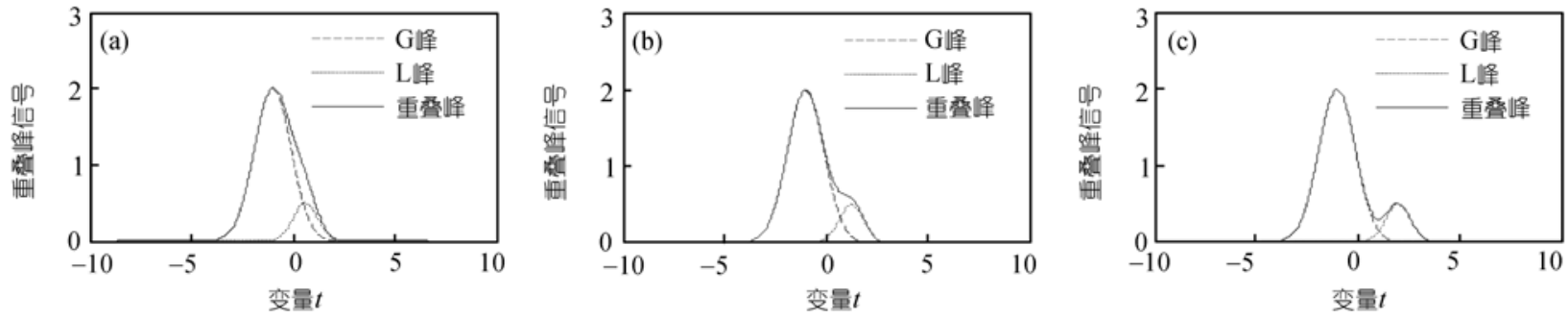

图 4 不同分离度的仿真重叠峰

表 1 处理前后 3 种 Gaussian 和 Lorentzian 重叠峰信号的峰位置、峰高、峰宽及其误差

\begin{tabular}{|c|c|c|c|c|c|c|c|c|c|}
\hline \multirow{2}{*}{ 混合双峰 } & \multicolumn{3}{|c|}{ 处理前 } & \multicolumn{3}{|c|}{ 处理后 } & \multicolumn{3}{|c|}{ 相对误差/\% } \\
\hline & 峰位 $\mu$ & 峰高 A & 峰宽 $\sigma$ & 峰位置 $\mu$ & 峰高 $A$ & 峰宽 $\sigma$ & 峰位 & 峰高 & 峰宽 \\
\hline \multirow[t]{2}{*}{$R=0.5000$} & -1.0 & 2.0 & 0.9 & -0.9977 & 2.0330 & 0.8989 & 0.2270 & 1.6494 & 0.1247 \\
\hline & 0.5 & 0.5 & 0.6 & 0.5156 & 0.4904 & 0.5892 & 3.1121 & 1.9161 & 1.7932 \\
\hline \multirow[t]{2}{*}{$R=0.7500$} & -1.0 & 2.0 & 0.9 & -1.0069 & 2.0141 & 0.8960 & 0.6892 & 0.7030 & 0.4461 \\
\hline & 1.25 & 0.5 & 0.6 & 1.2598 & 0.5049 & 0.5911 & 0.7813 & 0.9861 & 1.4869 \\
\hline \multirow[t]{2}{*}{$R=1.0000$} & -1.0 & 2.0 & 0.9 & -1.0098 & 2.0065 & 0.8950 & 0.9807 & 0.3228 & 0.5589 \\
\hline & 2.0 & 0.5 & 0.6 & 2.0110 & 0.5015 & 0.5906 & 0.5502 & 0.2900 & 1.5705 \\
\hline
\end{tabular}
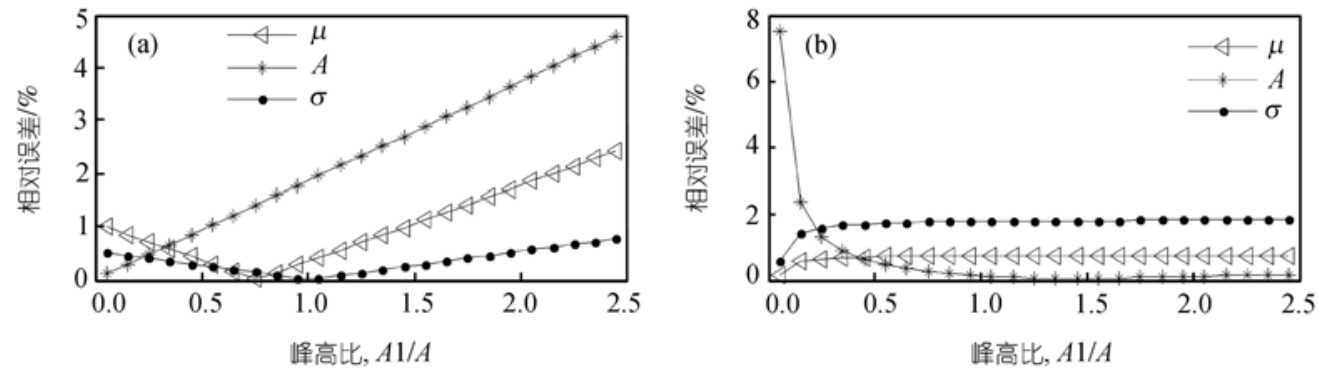

图 5 峰高比对参数估计的影响

(a) 峰高比对 Gaussian 峰参数估计误差的影响; (b) 峰高比对 Lorentzian 峰参数估计误差的影响

以 Gaussian 和 Lorentzian 混合双峰为例, 在给定 Gaussian 峰参数 $\mu=-1.0, A=20, \sigma=0.5$ 和 Lorentzian 峰参数 $\mu 1=0.5, \mathrm{~A} 1=1.0, \sigma 1$ 由峰宽比确定, 考察峰宽 比对参数估计误差的影响, 其结果见图 6 .

以 Gaussian 和 Lorentzian 混合双峰为例, 在给定 Gaussian 峰参数 $\mu=1.0, A=5, \sigma=2.0$ 和 Lorentzian 峰 参数 $A 1=1, \sigma 1=1, \mu 1$ 由分离度 $R$ 确定, 考察分离度对 参数估计误差的影响, 其结果见图 7 .

由于测量数据不可避免的带有误差, 而微分运 算对噪声又很敏感, 因此需要对数据进行预处理, 针 对分布信号的特征，采用以下几个指标来评价滤波 性能：（1）峰位置偏差 $\Delta P=|P-\widehat{P}| ; \quad$ （2）峰高失真 $\Delta H=|H-\hat{H}| / H \times 100 ; \quad$ (3) 峰宽失真 $\Delta W=|W-\widehat{W}| /$
$W \times 100 ;(4)$ 除噪度 $R=\sigma^{2} / \widehat{\sigma}^{2}$; 式中 $P, H, W, \sigma$ 分 别为原始分布信号的峰位置、峰高、峰宽和方差, $\hat{P}$, $\hat{H}, \widehat{W}, \hat{\sigma}$ 分别为消噪后的分布信号的峰位置、峰 高、峰宽和方差. 其中前三个指标用来评价除噪后信 号的失真程度，最后一个指标用来评价噪声的抑制 效果. 为了选择合适的消噪方法, 我们分别采用移动 平均法、多项式拟合法、小波滤波法以及小波加多项 式拟合的方法对分布信号进行消噪处理, 然后通过 上述 4 个指标的对比，最后选择小波加多项式拟合法 消噪. 以 Gaussian 和 Lorentzian 混合双峰为例, 在给 定 Gaussian 峰参数 $\mu=-1.5, A=5, \sigma=1.2$ 和 Lorentzian 峰参数 $\mu 1=1.5, A 1=1.0, \sigma 1=0.8$, 考察信噪比对参数 估计误差的影响, 其结果见图 8. 

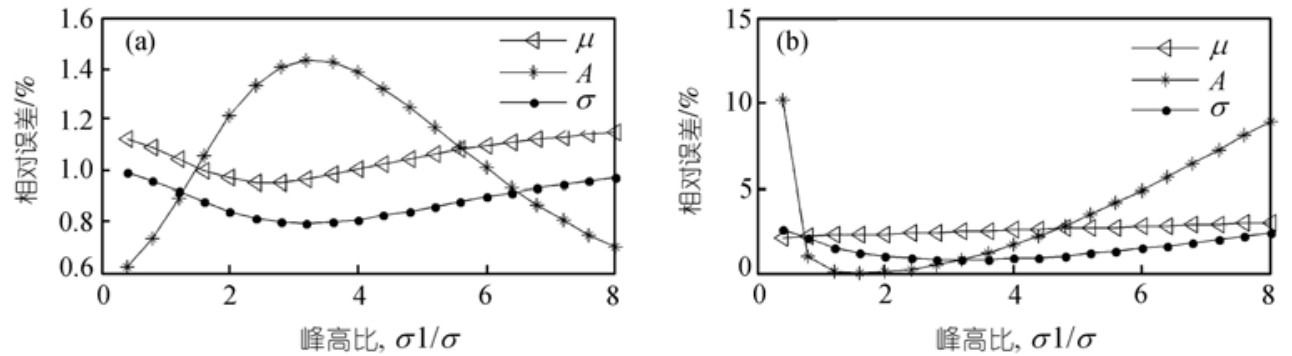

图 6 峰宽比对参数估计的影响

(a) 峰宽比对 Gaussian 峰参数估计误差的影响; (b) 峰宽比对 Lorentzian 峰参数估计误差的影响
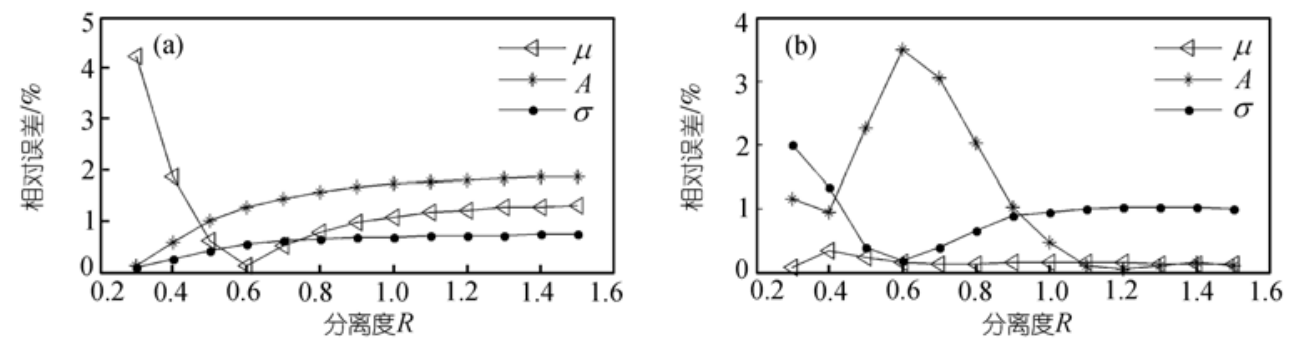

图 7 分离度对参数估计误差的影响

(a) 分离度对 Gaussian 峰参数估计误差的影响; (b) 分离度对 Lorentzian 峰参数估计误差的影响
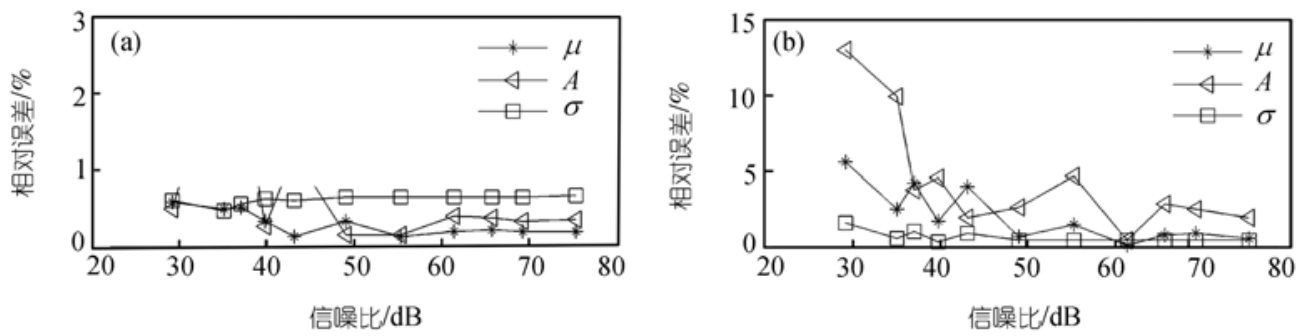

图 8 信噪比对参数估计误差的影响

(a) 信噪比对 Gaussian 峰参数估计误差的影响; (b) 信噪比对 Lorentzian 峰参数估计误差的影响 高斯峰参数为: $\mu=-1.5, A=5, \sigma=1.2$; Lorentzian 峰参数为: $\mu=1.5, A 1=1.0, \sigma=0.8$, 分离度 $R=0.75$

\section{2 用 Tsallis 峰拟合已知重叠峰}

已知重叠峰信号 $f=f_{1}+f_{2}$, 其中 $f_{1}(t)=A e^{\left[-(t-\mu)^{2} /\left(2 \sigma^{2}\right)\right]}$ 为 Gaussian 峰, 另一个为 Lorentzian 峰 $f_{2}(t)=A \sigma^{2}$ / $\left[(t-\mu)^{2}+\sigma^{2}\right]$, 它们的参数分别为: $\mu=1.5, A=5.0$, $\sigma=1.2$ 和 $\mu=1.5, A=2.0, \sigma=0.8,-10 \leqslant t \leqslant 10$. 重叠峰 信号 $f$ 见图 9(a).

Gaussian 峰信号和 Lorentzian 峰信号的估计参数 见表 2. 为了评价估计效果, 用参数分别为: -1.4933 , 4.9998, 1.1964, 1.01 和 1.4926, 2.0093, 0.8241, 2.07 的 Tsallis 峰去拟合重叠峰信号, 其结果见图 9(b). 其
最大拟合相对误差为 $0.0052 \%$. 此外，从表 3 中可看 出, 通过扫描参数 $q$ 的办法可以找到拟合重叠峰信号 的参数 $q$, 以及其他相应参数.

\section{3 用 Tsallis 分布拟合实际重叠峰}

将实验所采集的数据输入到微机中进行处理, 记录所得的伏安信号如图 10(a)所示, 可以看出, 由 于铟和镉的还原电位很靠近, 造成它们的伏安峰严 重重叠, 因此很难用 DPV 直接进行它们的同时测定. 而这样重叠的伏安峰经本文方法处理的结果见图 10(b). 可以看出, 经处理后原本难以测定的峰高、峰 电位和峰宽现在已被准确地提取出来. 峰位置、峰高 

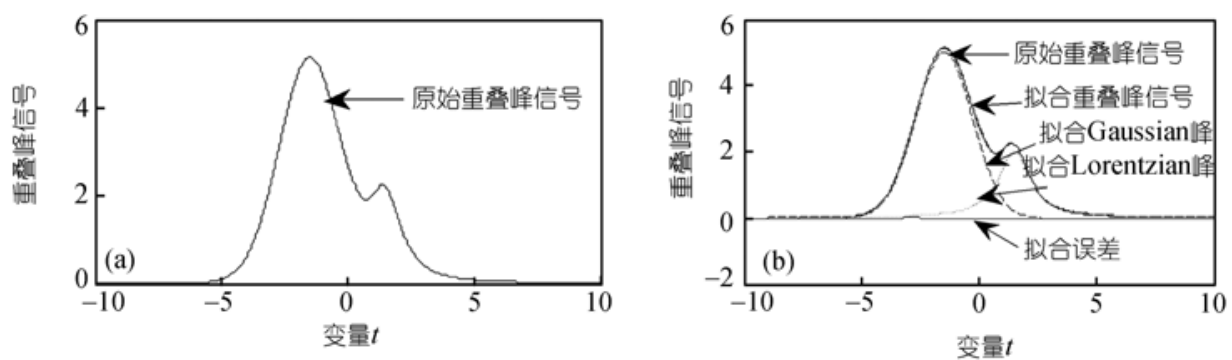

图 9 用 Tsallis 峰拟合重叠峰结果

(a) 原始信号; (b) 拟合结果

表 2 已知重叠峰信号的参数估计结果

\begin{tabular}{|c|c|c|c|c|c|c|c|c|c|}
\hline \multirow{2}{*}{ 峰 } & \multicolumn{3}{|c|}{ 原始参数 } & \multicolumn{3}{|c|}{ 估计参数 } & \multicolumn{3}{|c|}{ 相对误差\% } \\
\hline & 峰位 $\mu$ & 峰高 $A$ & 峰宽 $\sigma$ & 峰置 $\mu$ & 峰高 $A$ & 峰宽 $\sigma$ & 峰位置 & 峰高 & 峰宽 \\
\hline Gaussian 峰 & -1.5 & 5.0 & 1.2 & -1.4933 & 4.9998 & 1.1964 & 0.4496 & 0.0039 & 0.3031 \\
\hline Lorentzian 峰 & 1.5 & 2.0 & 0.8 & 1.4926 & 2.0093 & 0.8241 & 0.4931 & 0.4670 & 3.0099 \\
\hline
\end{tabular}

表 3 用 Tsallis 峰拟合已知重叠峰信号确定参数 $q$ 的过程

\begin{tabular}{cccccc}
\hline \multirow{2}{*}{$q$} & \multicolumn{2}{c}{ Gaussian 峰 } & & \multicolumn{2}{c}{ Lorentzian 峰 } \\
\cline { 2 - 3 } & 估计式 I 得到的 $\sigma$ & 估计式 II 得到的 $\sigma$ & & 估计式 I 得到的 $\sigma$ & 估计式 II 得到的 $\sigma$ \\
\hline 0.98 & 1.1826 & 1.2286 & 2.01 & 0.7913 & 0.8025 \\
0.99 & 1.1886 & 1.2157 & 2.02 & 0.7966 & 0.8058 \\
1.00 & 1.1945 & 1.2039 & 2.03 & 0.8021 & 0.8092 \\
$\mathbf{1 . 0 1}$ & $\mathbf{1 . 2 0 0 5}$ & $\mathbf{1 . 1 9 2 2}$ & 2.04 & 0.8076 & 0.8127 \\
1.02 & 1.2065 & 1.1815 & 2.05 & 0.8131 & 0.8163 \\
1.03 & 1.2126 & 1.1715 & 2.06 & 0.8188 & 0.8199 \\
1.04 & 1.2187 & 1.1621 & 2.07 & $\mathbf{0 . 8 2 4 5}$ & $\mathbf{0 . 8 2 3 6}$ \\
1.05 & 1.2248 & 1.1532 & 2.08 & 0.8303 & 0.8274 \\
1.06 & 1.2309 & 1.1449 & 2.09 & 0.8362 & 0.8313 \\
\hline
\end{tabular}
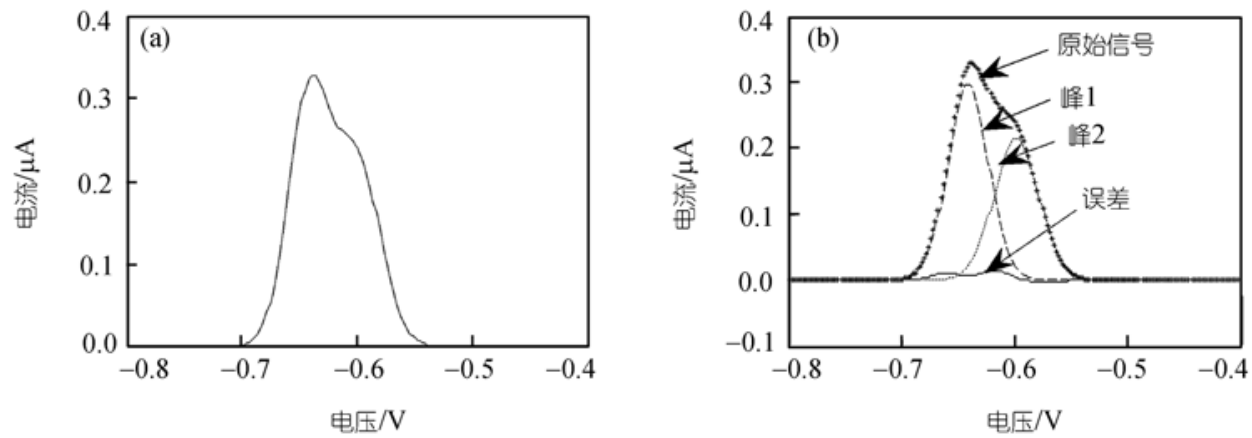

图 $10 \mathrm{Cd}(\mathrm{II})$ 和 $\mathrm{In}(\mathrm{III})$ 的混和体系原始方波伏安信号(a)与采用本文方法处理的结果(b)

表 4 本文方法提取的 Cd( II ) 和 In(III)峰位、峰高、峰宽的结果

\begin{tabular}{|c|c|c|c|c|c|c|c|c|c|}
\hline \multirow{2}{*}{ 体系 } & \multicolumn{3}{|c|}{ 处理前 ${ }^{\text {a) }}$} & \multicolumn{3}{|c|}{ 处理后 } & \multicolumn{3}{|c|}{ 相对误差/\% } \\
\hline & 峰位置 $\mu$ & 峰高 $A$ & 峰宽 $\sigma$ & 峰位置 $\mu$ & 峰高 $A$ & 峰宽 $\sigma$ & 峰位置 & 峰高 & 峰宽 \\
\hline In(III) & -0.642 & 0.300 & 0.018 & -0.6415 & 0.2979 & 0.0176 & 0.0760 & 0.6927 & 2.1043 \\
\hline $\mathrm{Cd}(\mathrm{II})$ & -0.600 & 0.220 & 0.020 & -0.5989 & 0.2154 & 0.0196 & 0.1850 & 2.0834 & 1.9407 \\
\hline
\end{tabular}

a) 处理前的各数据为各离子单独存在于溶液中所测的电位, 以其作为理论电位计算其相对误差 
和峰宽的误差见表 4. 采用本文的方法, 不但可得到 重叠峰的峰位置，还可同时得到峰高和峰宽这两个 参数, 将传统峰分辨和峰分解两个步骤合二为一. 从 处理结果来看, 就峰位相对误差, 本文的结果要比文 献[7,8]要好, 而且它的峰高和峰宽的相对误差都小 于 3\%, 这表明了该方法的有效性和可靠性.

\section{5 结论}

借助于分数阶微分工具, 给出了一种新的重叠 峰分离方法, 建立了 3 种分布信号的微分的过零点与 其相应的微分阶次的估计模型 I 和极值与其相应的 微分阶次的估计模型 II，分析了峰高比、峰宽比、分 离度和噪声对参数估计结果的影响. 采用本文方法不 但能保留导数法分峰的优点, 还能同时得到各子峰的 特征参数, 将传统峰分辨中的确定子峰位置和分解 子峰两个步骤合二为一, 简化了分析过程.

\section{参考文献}

1 卢小泉, 刘宏德, 张敏, 王晓强, 薛中华, 康敬万. 分数导数结 合傅里叶最小二乘拟合处理含噪音的重迭信号. 分析化学, 2003, 33(2): 143-147

2 Sten O E. The Fourier transform of voltammnetric peak and its use in resolution enhancement. J Electroanal Chem, 1990, 296: 371-
394

3 Biserka R, Ivanka P, Marko R. Comparative quantitative analysis of overlapping voltammetric signals. Anal Chem Acta, 1994, 285: 103-111[DOI]

4 方建兴, 吴茂成, 王定兴. 微分消卷积法提高重叠谱图的分辨 率. 光谱学与光谱分析, 1998, 18(6): 666-668

5 王瑛, 莫金垣, 陈晓燕. 二阶样条小波卷积法分辨重叠化学信 号. 中国科学 B 辑: 化学, 2003, 33(04): 296-305

6 邱建丁, 梁汝萍, 邹小勇, 莫金垣. 小波分形分析重叠峰信号. 分析测试学报, 2003, 22(5): 1-5

7 郑小萍, 莫金垣, 蔡沛祥. 样条小波变换用于分辨重叠伏安峰 的研究. 中国科学 B 辑: 化学, 1999, 29(02): 141-147

8 郑建斌, 赵瑞, 张红权等. 小波变换及其在化学中的应用. 分析 化学, 1999, 27(7): 855-861

9 邹小勇, 莫金垣, 样条小波重叠峰分析. 科学通报, 1999, 48(8): 818

10 邵学广, 庞春艳, 孙莉. 小波变换与化学信号处理. 化学进展, 2000, 12(3): 233-244

11 李一波, 黄小原. 基于 RBFNN 和 GA 的重叠峰分辨技术. 应用 科学学报, 2002, 20(1): 99-103

12 Huag W, Henderson T L E, Bond A M, Oldham K B. Curve fitting to resolve overlapping voltammetric peaks: model and examples. Analytica Chimica Acta, 1995, 304(1): 1-15

13 Butzer P L, Westphal U. An Introduction to Fractional Calculus. Singapore: World Scientific, 2000. 1-85

14 聂否. 小波变换用于重叠化学信号的分辨研究. 博士学位论文. 合肥：中国科学技术大学, 2002. 16-17 